NKU-2017-SF2

\title{
Charged dilaton black hole in $2+1$ dimensions as a particle accelerator
}

\author{
Sharmanthie Fernando 1 \\ Department of Physics, Geology \& Engineering Technology \\ Northern Kentucky University \\ Highland Heights \\ Kentucky 41099 \\ U.S.A.
}

\begin{abstract}
In this paper we have studied particle collisions around a charged dilaton black hole in $2+1$ dimensions. This black hole is a solution to the low energy string action in $2+1$ dimensions. Time-like geodesics for charged particles are studied in detail. The center of mass energy for two charged particles colliding closer to the horizon is calculated and shown to be infinite if one of the particles has the critical charge.
\end{abstract}

Key words: charged, accelerator, black hole, dilaton, strings

\section{Introduction}

Banãdos, Silk and West [1] demonstrated that two particles colliding near the horizons of an extreme Kerr black could create large center of mass (CM) energy if one of the particles has critical angular momentum. This process is known as the BSW mechanism and has drawn many to study black holes in this context. Scattering energy of the particles in the vicinity of a rotating black hole can reach very large values for non extreme rotating black holes as demonstrated in [2]. The same is true for the Kerr-de Sitter black hole [3]. BSW effect of the extreme Kerr-Taub-NUT space-time has been studied by Liu et.al [4], of a rotating black hole in string theory (Sen black hole) were studied by Wei et.al. [5], and of a rotating cylindrical black hole was studied in [6].

Non rotating charged black holes also could act as particle accelerators when they are extreme as pointed out by Zaslavskill [7]. This phenomena where extreme charged black holes with charged particles acting as particle accelerators works for string black holes was shown by Fernando in [8] and for Einstein-Maxwell-dilaton black holes in

\footnotetext{
${ }^{1}$ fernando@nku.edu
} 
[9]. Acceleration of particles also could occur inside spinning black holes as shown in [10].

Particle acceleration studies also have been extended to regular black holes with a non-singular center: Rotating Hayward regular black hole as a particle accelerator was studied by Amir and Ghosh [11. Rotating Ayòn-Beato-Garcia black hole which is also regular was studied in this context in [12] and the rotating Bardeen black hole was studied as a particle accelerator in [13]. An excellent review on black holes as particle accelerators is written by Harada [14]. Collisions of spinning particles also can create infinite center of mass energy as described in [15] [16] [17].

In this paper our goal is to study particle acceleration in a charged dilaton black hole in $2+1$ dimensions. Black holes in three dimensions originated from the well known BTZ black hole [18] [19] which is a black hole with a negative cosmological constant. The BTZ black hole has attracted lot of attention since many aspects of black holes can be studied in a simpler setting. The dilaton black hole considered in this paper was derived by Chan and Mann [20]. This particular black hole is important in the sense that it is a solution to low energy string theory in $2+1$ dimensions. There are several extensions for dilaton black holes in $2+1$ dimensions: New class of dilaton solutions were generated by applying T-dulity in [21]. Rotating dilaton solutions were generated by compactifying a 4D cylindrical solutions by Fernando [22]. Dilaton black holes with non-linear electrodynamics were derived by Hendi et.al [23].

As far as particle acceleration is considered, there are very few works in low dimensions. Particle acceleration by the BTZ black hole was studied by Yang et. al [24], by the charged hairy black holes was studied by Sadeghi et.al. [25], by the spinning dilaton black hole was studied by Fernando [26].

The paper is organized as follows: in section 2, we will present the details of the charged dilaton black hole in $2+1$ dimensions. In section 3 , time-like geodesics for the charged dilaton black hole are presented. The effective potential is analyzed in section 4 and the center of mass is discussed in section 5. Finally the conclusion is given in section 6 .

\section{Introduction to the charged dilaton black hole in $2+1$ dimensions}

The charged black hole considered in this paper was derived by Chan and Mann [20] for the following action,

$$
S=\int d^{3} x \sqrt{-g}\left[R-\frac{B}{2}(\nabla \phi)^{2}-e^{-4 \alpha \phi} F_{\mu \nu} F^{\mu \nu}+2 e^{\beta \phi} \Lambda\right]
$$

Here $\Lambda$ is the cosmological constant where $\Lambda>0$ represents anti-de Siter and $\Lambda<0$ represents de-Sitter space. 
The constants $\alpha, \beta$ and $B$ are arbitrary couplings, $\phi$ is the dilaton field, $R$ is the scalar curvature and $F_{\mu \nu}$ is the Maxwell's field strength. A family of black hole solutions with rotational symmetry for the above action were obtained as,

$$
\begin{aligned}
& d s^{2}=-\left(-2 M r^{\frac{2}{N}-1}+\frac{8 \Lambda r^{2}}{(3 N-2) N}+\frac{8 Q^{2}}{(2-N) N}\right) d t^{2} \\
& +\frac{4 r^{\frac{4}{N}-2} d r^{2}}{N^{2} \gamma^{4 / N}\left(\left(-2 M r^{2 / N-1}+\frac{8 \Lambda r^{2}}{(3 N-2) N}+\frac{8 Q^{2}}{(2-N) N}\right)\right.}+r^{2} d \theta^{2}
\end{aligned}
$$

where,

$$
k= \pm \sqrt{\frac{N(2-N)}{2 B}} ; \quad 4 \alpha k=\beta k=N-2 ; \quad 4 \alpha=\beta ;
$$

The dilaton field is given by

$$
\phi=\frac{2 k}{N} \ln \left(\frac{r}{r_{0}}\right)
$$

In this paper we will focus on a special class of black holes with $N=1, k=$ $-\frac{1}{4}, \beta=4 \alpha=4$. In this case, the action in eq(1) is conformally coupled to the low energy string effective action in $2+1$ dimensions. The black hole solution in this particular case is given by,

$$
d s^{2}=-f(r) d t^{2}+\frac{1}{h(r)}+r^{2} d \theta^{2}
$$

where,

$$
f(r)=\left(-2 M r+8 \Lambda r^{2}+8 Q^{2}\right)
$$

and

$$
h(r)=\frac{\left(-2 M r+8 \Lambda r^{2}+8 Q^{2}\right)}{4 r^{2}}
$$

The dilaton and the electric field are given by,

$$
\phi=-\frac{1}{2} \ln \left(\frac{r}{r_{0}}\right) ; \quad F_{r t}=\frac{Q}{r^{2}}
$$

The above space-time has two horizons if $M \geq 8 Q \sqrt{\Lambda}$ and they are given by,

$$
r_{h}=\frac{M+\sqrt{M^{2}-64 Q^{2} \Lambda}}{8 \Lambda} ; \quad r_{c}=\frac{M-\sqrt{M^{2}-64 Q^{2} \Lambda}}{8 \Lambda}
$$

Here $r_{h}$ corresponds to the event horizon and $r_{c}$ corresponds to the Cauchy horizon. The two horizons merge to give an extreme black hole when $M=8 Q \sqrt{\Lambda}$. There is a time-like singularity at $r=0$. The Hawking temperature $T_{\text {Hawking }}$ is given by,

$$
T_{\text {Hawking }}=\left.\frac{1}{4 \pi}\left|\frac{d g_{t t}}{d r}\right| \sqrt{-g^{t t} g^{r r}}\right|_{r=r_{h}}=\frac{M}{4 \pi r_{h}} \sqrt{1-\frac{64 Q^{2} \Lambda}{M^{2}}}
$$


There are several works on the charged dilaton black hole presented above: Quasinormal modes of charged and neutral scalar fields were studied by Fernando [27], [28] and by Myung et.al. [29]. Grey body factors of neutral scalar fields were studied in [30]. Geodesics of the black hole were studied by Fernando et.al. in [31].

\section{Time-like geodesics of the test particles of the charged dilation black hole}

In this section we will present time-like geodesics of a test particle with charge per unit mass $e$ around the black hole considered in this paper. We will follow the formalism in Chandrasekhar's book [32. The Lagrangian of the charged test particle in this black hole back ground is given by,

$$
\mathcal{L}=-\frac{1}{2}\left(g_{\mu \nu} \frac{d x^{\mu}}{d \tau} \frac{d x^{\nu}}{d \tau}\right)+e A_{\mu} \frac{d x^{\mu}}{d \tau}
$$

Here the parameter $\tau$ is the proper time of the charged particle. The metric functions and $A_{\mu}$ are given by,

$$
g_{t t}=-f(r) ; \quad g_{r r}=\frac{1}{h(r)} ; \quad g_{\phi \phi}=r^{2} ; \quad A_{t}=\frac{Q}{r}
$$

Now, the Lagrangian can be written explicitly as,

$$
L=-\frac{1}{2}\left(-f(r)\left(\frac{d t}{d \tau}\right)^{2}+\frac{1}{h(r)}\left(\frac{d r}{d \tau}\right)^{2}+r^{2}\left(\frac{d \phi}{d \tau}\right)^{2}-2 \frac{e Q}{r}\left(\frac{d t}{d \tau}\right)\right)
$$

Each coordinate in the space-time has a corresponding canonical momenta $p_{\mu}$ which can be obtained from the Lagrangian as,

$$
\begin{gathered}
p_{t}=\frac{\partial L}{\partial \dot{t}}=f(r) \dot{t}+\frac{e Q}{r} \\
p_{\phi}=-\frac{\partial L}{\partial \dot{\phi}}=r^{2} \dot{\phi} \\
p_{r}=-\frac{\partial L}{\partial \dot{r}}=\frac{\dot{r}}{h(r)}
\end{gathered}
$$

The charged dilaton black hole has two Killing vectors $\partial_{t}$ and $\partial_{\phi}$. Hence, the canonical momenta $p_{t}$ and $p_{\phi}$ are conserved and these constants are labeled as energy per unit mass $E$ and angular moment per unit mass $L$. From eq.(14) and eq.(15), $E$ and $L$ can be solved to be,

$$
E=f(r) \dot{t}+\frac{e Q}{r}
$$




$$
L=r^{2} \dot{\phi}
$$

Eq(17) and eq(18) can be solved to obtain $\dot{t}$ and $\dot{\phi}$ as,

$$
\begin{gathered}
\dot{t}=\frac{\left(E-\frac{e Q}{r}\right)}{f(r)}=u^{t} \\
\dot{\phi}=\frac{L}{r^{2}}=u^{\phi}
\end{gathered}
$$

We will assume that $\dot{t}>0$ for all $r>r_{h}$. Hence the motion is forward in time outside the black hole event horizon. This implies,

$$
\left(E-\frac{e Q}{r}\right)>0, \quad \forall r>r_{h}
$$

The components of the velocity of the particle are given by $u^{\mu}=\frac{d x^{\mu}}{d \tau}$ and they are normalized as, $u^{\mu} u_{\mu}=-1$. The normalized condition is expressed as

$$
g_{t t}\left(u^{t}\right)^{2}+g_{r r}\left(u^{r}\right)^{2}+g_{\phi \phi}\left(u^{\phi}\right)^{2}=-1
$$

leading to,

$$
\dot{r}^{2}=\frac{-\left(1+g_{t t} \dot{t}^{2}+g_{\phi \phi} \dot{\phi}^{2}\right)}{g_{r r}}
$$

By substituting $u^{t}$ and $u^{\phi}$ from eq(19) and eq(20), one can obtain $u^{r}=\dot{r}$ as,

$$
\dot{r}^{2}=\frac{1}{4 r^{4}}\left(-f(r)\left(r^{2}+L^{2}\right)+(E r-e Q)^{2}\right)
$$

By identifying,

$$
K=(E r-e Q)
$$

and

$$
H^{2}=K^{2}-f(r)\left(r^{2}+L^{2}\right)
$$

$\dot{r}$ can be written in a short form as,

$$
\dot{r}=-\frac{H}{2 r^{2}}=u^{r}
$$

The minus sign for $\dot{r}$ is chosen since we assume the particle is falling towards the black hole. 


\section{Effective potential for the charged dilaton black hole}

In this paper our main motivation is to understand the collision near the horizon. Hence it is vital to see if the particle could reach the horizon. In order to achieve that we will study the effective potential for the charged particle given by the relation,

$$
\dot{r}^{2}+V_{e f f}=0
$$

From eq(24), one obtain $V_{\text {eff }}$ as,

$$
V_{e f f}=\frac{f(r)\left(r^{2}+L^{2}\right)-K^{2}}{4 r^{4}}
$$

For $r \rightarrow \infty, V_{\text {eff }} \rightarrow 2 \Lambda$ and for $r \rightarrow 0, V_{\text {eff }} \rightarrow \infty$. Since $\dot{r}=\sqrt{-V_{\text {eff }}}$, the particle motion is possible only in the regions where $V_{\text {eff }}<0$. Hence the particle should start the motion from a finite distance from the black hole. In Fig(1) and Fig(2), $V_{\text {eff }}$ and $f(r)$ are plotted. Fig.(1) represents a non-degenerate black hole and Fig(2) represents an extreme black hole. The effective potential has two roots for the given parameters. What is noteworthy is that the larger root lies outside the event horizon. At $r=r_{h}$,

$$
V_{e f f}=-\frac{\left(E r_{h}-e Q\right)^{2}}{4 r_{h}^{4}}=-\frac{K\left(r_{h}\right)^{2}}{4 r^{4}}
$$

Hence $V_{\text {eff }} \leq 0$ at the horizon. $V_{\text {eff }}=0$ for a critical charge $e_{c}$ given by,

$$
e_{c}=\frac{E r_{h}}{Q}=\frac{E}{Q}\left(\frac{M+\sqrt{M^{2}-64 Q^{2} \Lambda}}{8 \Lambda}\right)
$$

Notice that when $V_{\text {eff }}=0, K\left(r_{h}\right)=0$. We calculated the roots of $V_{\text {eff }}$ numerically and have plotted in Fig(3). It is clear that the root $r_{o} \geq r_{h}$ for all $e>e_{c}$. Therefore if $e>e_{c}$, the particle can start at rest from a finite distance from the horizon and fall towards the black hole. The larger the $e$, further away the particle can start its motion. 


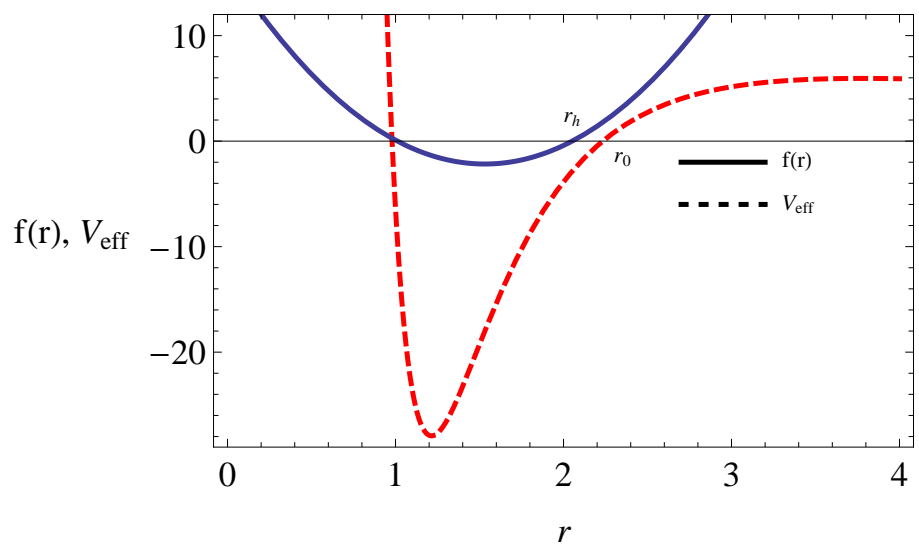

Figure 1: The figure shows $f(r)$ and $V_{\text {eff }}$ vs $r$. Here $M=12.25, Q=1.44, E=$ 7.7, $\Lambda=1, e=1.1$ and $L=11.5$

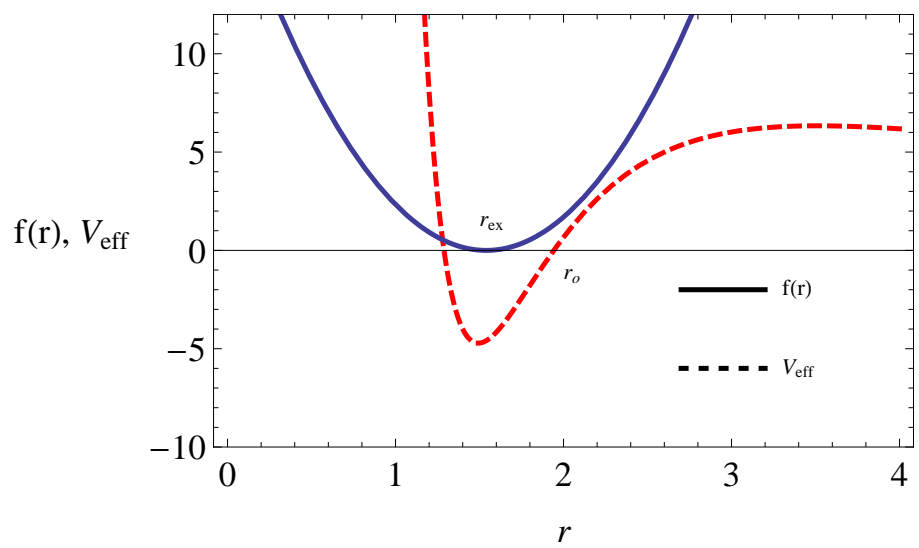

Figure 2: The figure shows $f(r)$ and $V_{\text {eff }}$ vs $r$ for an extreme black hole. Here $M=12.32, Q=1.54, E=7.7, \Lambda=1, e=1.1$ and $L=11.5$ 


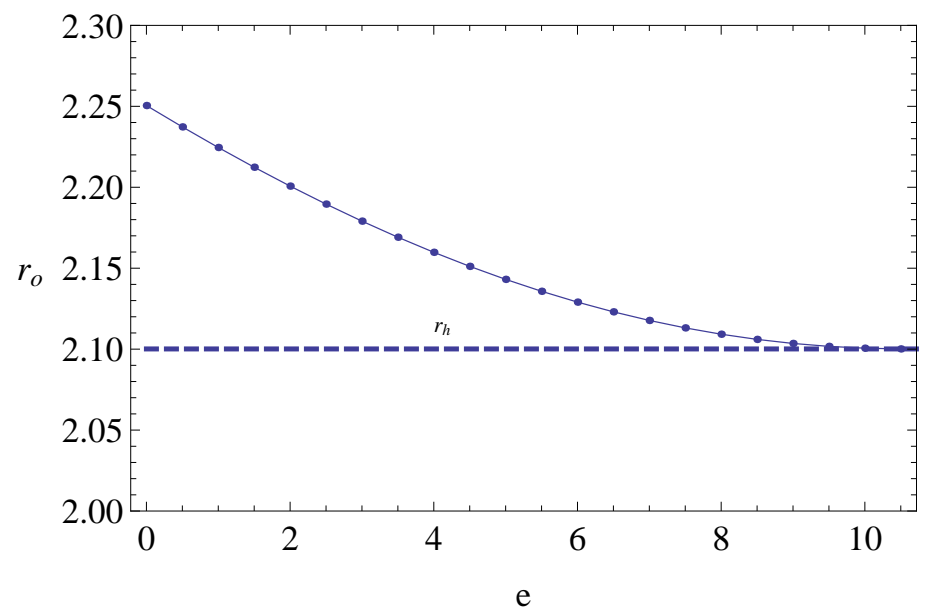

Figure 3: The figure shows $r_{0}$ vs $e$. Here $M=12.35, Q=1.44, E=7.6, \Lambda=1$ and $L=13.1$

\section{Collision of two charged particles and their cen- ter of mass energy}

In this section we will calculate the center of mass energy of two charged particles colliding closer to the horizon. The four velocities of the two particles are, $u_{1}^{\mu}$ and $u_{2}^{\mu}$. The rest mass of the particles are assumed to be equal and is given by $m_{0}$. Hence the center of mass energy is given by,

$$
\tilde{E}_{c m}=2 m_{0}^{2}\left(1-g_{\mu \nu} u_{1}^{\mu} u_{2}^{\nu}\right)
$$

In the rest of the paper we will calculate $E_{c m}=\tilde{E}_{c m} /\left(2 m_{0}^{2}\right)$ given by,

$$
E_{c m}=\frac{\left(f(r)\left(r^{2}-L_{1} L_{2}\right)-\left(K_{1} K_{2}-H_{1} H_{2}\right)\right)}{r^{2} f(r)}
$$

where,

$$
\begin{gathered}
K_{1}=r E_{1}-e_{1} Q \\
K_{2}=r E_{2}-e_{2} Q \\
H_{1}=K_{1}^{2}-f(r)\left(L_{1}^{2}+r^{2}\right) \\
H_{2}=K_{2}^{2}-f(r)\left(L_{2}^{2}+r^{2}\right)
\end{gathered}
$$

When the particles reach the horizon with $r=r_{h}, f(r) \rightarrow 0, H_{1} \rightarrow \sqrt{K_{1}^{2}}$, and $H_{2} \rightarrow \sqrt{K_{2}^{2}}$. Therefore,

$$
E_{c m}\left(r \rightarrow r_{h}\right)=\frac{1}{r_{h}^{2} f\left(r_{h}\right)}\left(K_{1} K_{2}-\sqrt{K_{1}^{2}} \sqrt{K_{2}^{2}}\right)
$$


Since $f\left(r_{h}\right)=0$, the denominator of the above expression is zero. Also, due to the condition given in eq(21) $, K_{1}\left(r_{h}\right), K_{2}\left(r_{h}\right) \geq 0$. Hence the numerator is also zero leading to an undetermined value for the center of mass energy at the horizon. However, one can use the L' Hospital's rule to calculate the limiting value of $E_{c m}(r \rightarrow$ $r_{h}$ ) resulting,

$$
E_{c m}=\frac{A_{1} r_{h}^{4}+A_{2} r_{h}^{3}+A_{3} r_{h}^{2}+A_{4} r_{h}+A_{5}}{2 r_{h}^{2} K_{1}\left(r_{h}\right) K_{2}\left(r_{h}\right)}
$$

where

$$
\begin{gathered}
A_{1}=-\left(E_{1}-E_{2}\right)^{2} \\
A_{2}=2\left(e_{1}-e_{2}\right)\left(E_{1}-E-2\right) Q \\
A_{3}=-\left(E_{1} L_{2}+E_{2} L_{1}\right)^{2}-\left(e_{1}-e_{2}\right)^{2} Q^{2} \\
A_{4}=2 Q\left(e_{2} L_{1}+e_{1} L_{2}\right)\left(E_{2} L_{1}+E_{1} L_{2}\right) \\
A_{5}=-Q^{2}\left(e_{2} L_{1}+e_{1} L_{2}\right)^{2}
\end{gathered}
$$

The numerator of eq(39) is finite. So, if $K_{1}\left(r_{h}\right)=0$ or $K_{2}\left(r_{h}\right)=0$, the center of mass energy becomes infinity. When one solve $K_{1}\left(r_{h}\right)=0$ or $K_{2}\left(r_{h}\right)=0$, one obtain the critical charge $e_{c 1}$ or $e_{c 2}$ which was discussed in section(4). We have plotted $E_{c m}$ in Fig(4) for varying $e_{1}$ with all other parameters fixed. When particle number 1 reach the critical charge $e_{1} c, E_{c m}$ goes to infinity. Notice that the critical charge has to be reached from the left of the asymptotic value in order to get a positive value for the center of mass energy.

It is important to notice that to achieve high values of $E_{c m}$, only one of the particles should have the critical charge. If both have the critical charges, then $E_{c m}$ will be finite. One can prove it as follows: When both particles have critical charge, both $K_{1}\left(r_{h}\right)=K_{2}\left(r_{h}\right)=0$. This implies that $H_{1}\left(r_{h}\right)=H_{2}\left(r_{h}\right)=0$. Hence for this special case,

$$
E_{C M}=\frac{\left(r_{h}^{2}-L_{1} L_{2}\right)}{r_{h}^{2}}=1-\frac{L_{1} L_{2}}{r_{h}^{2}}
$$

which is finite. 




Figure 4: The figure shows $E_{c m}$ vs $e_{1}$. Here $M=10, Q=1, \Lambda=1, E_{1}=1.14, E_{2}=$ $0.725, L_{1}=0.4, L_{2}=0.3$

\section{Conclusions}

In this paper we have studied particle collisions around a charged dilaton black hole in $2+1$ dimensions. This black hole is a solution to the low energy string theory in $2+1$ dimensions. The given black hole has two horizons which depend on $M, Q$ and $\Lambda$. If $M=8 Q \sqrt{\Lambda}$, it has degenerate horizon.

The time-like geodesics for charged particles are studies in detail. The three velocities, $u^{t}, u^{r}, u^{\phi}$ are calculated. The effective potential reaches $2 \Lambda$ for large $r$ and goes to infinity for $r \rightarrow 0$. When the parameters are such that there are horizons, the effective potential has a root $r_{0}$ larger than the event horizon $r_{h}$. Hence a particle could start the motion at rest with $r<r_{0}$ and fall into the black hole. There is a critical charge at which $r_{0}=r_{h}$.

The main goal in this paper was to study the particle collision around the black hole. We found that the center of mass energy of two charged particles colliding will becomes infinity if one of the charged particles has the critical charge. Hence the charged dilaton black hole can be a particle accelerator.

\section{References}

[1] M. Banãdos, J. Silk, and S. M. West, Kerr black holes as particle accelerators to arbitrarily high energy, Phys. Rev. Lett. 103111102 (2009)

[2] A. A. Grib, and Yu. V. Pavlov, On the collisions between particles in the vicinity of rotating black holes, JETP Letters 92 (2010) 125 
[3] Y. Li, J. Yang, Y. Li, S. Wei, and Y. Liu, Particle acceleration in Kerr-(anti)-de Sitter black hole backgrounds, Class. Quant. Grav. 28225006 (2011)

[4] C. Liu, S. Chen, C. Ding, and J. Jing, Particle acceleration on the background of the Kerr-Taub-NUT space-time, Phys. Lett. B 701285 (2011)

[5] S. Wei, Y. Liu, H. Li, and F. Chen, Particle collisions on stringy black hole background, JHEP 1012066 (2010)

[6] J. L. Said, and K. Z. Adami, Rotating charged cylindrical black holes as particle accelerators, Phys. Rev. D 83104047 (2011)

[7] O. B. Zaslavskii, Acceleration of particles by non rotating charged black holes, Jour. Exp. Theo. Phys. Lett. 92571 (2010)

[8] S. Fernando, String black hole: can it be a particle accelerator ?, Gen. Rel. Grav. 461634 (2014)

[9] P. Mao, R. Li, L. Jia, and J. Ren, Acceleration of particles in Einstein-Maxwelldilaton black hole, arXiv:1008.2660

[10] K. Lake, Particle accelerators inside spinning black holes, Phys. Rev. Lett. 104 211102 (2010); Erratum: Phys. Rev. Lett. 104259903 (2010)

[11] M. Amir, and S. G. Ghosh, Rotating Hayward's regular black hole as particle accelerator, JHEP 1507015 (2015)

[12] S. G. Ghosh, P. Sheoran, and M. Amir, Rotating Ayòn-Beato-Garcia black hole as a particle accelerator, Phys. Rev. D 90103006 (2014)

[13] S. G. Ghosh, and M. Amir, Horizon structure of rotating Bardeen black hole and particle acceleration, Eur. Phy. Jou. C 75553 (2015)

[14] T. Harada, and M. Kimura, Black holes as particle accelerators: a brief review, Class. Quant. Grav. 31243001 (2014)

[15] O. B. Zaslavskii, Schwarzschild black hole as particle accelerator of spinning particles, Europhys. Lett. 11430003 (2016)

[16] M. Guo, and S. Gao, Kerr black holes as accelerators of spinning test particles, Phys. Rev. D 93084025 (2016)

[17] Y. Zhang, B. Gu, S. Wei, J. Yang, and Y. Liu, Charged spinning black holes as accelerators of spinning particles, Phys. Rev. D 94124017 (2016) arXiv:1608.08705

[18] M. Bañados, C. Teitelboim, and J. Zanelli, The Black Hole in Three Dimensional Space Time Phys. Rev. Lett. 691849 (1992) 
[19] M. Bañados, M. Henneaux, C. Teitelboim, and J. Zanelli, Geometry of the 2+1 Black Hole Phys. Rev. D 481506 (1993)

[20] K. C. K. Chan, and R. B. Mann, Static charged black holes in 2+1 dimensional dilaton gravity, Phys. Rev. D 50 (1994) 6385; erratum, D 522600 ( 1995)

[21] C. Chen, T-Duality and Spinning Solutions in $2+1$ Gravity, Nucl. Phys. B 544 775 (1999)

[22] S. Fernando, Rotating Dilaton Solutions in 2+1 Dimensions, Gen. Rel. Grav. 34 $461(2002)$

[23] S. H. Hendi, B. E. Panah, S. Panahiyan, and A. Sheykhi, Dilatonic BTZ black holes with power-law field, Phys. lett. B 767214 (2017)

[24] J. Yang, Y. Li, Y Li, S. Wei, and Y Liu, Particle collisions in the lower dimensional rotating black hole space-time with the cosmological constant, Adv. High. Energy. Phys. 2014204016 (2014)

[25] J. Sadeghi, B. Pourhassan, and H. Farahani, Rotating charged hairy black hole in $(2+1)$ dimensions and particle acceleration, Commun. Theor. Phys. 62358 (2014)

[26] S. Fernando, Spinning dilaton black hole in $2+1$ dimensions as a particle accelerator, arXiv:1703.00373 (To be published in Modern Physics Letters A)

[27] S. Fernando, Quasinormal modes of charged scalars around dilaton black Holes in 2+1 dimensions: exact frequencies, Phys. Rev. D 77124005 (2008)

[28] S. Fernando, Quasinormal modes of charged dilaton black holes in $2+1$ dimensions, Gen. Rel. Grav. 3671 (2004)

[29] Y. S. Myung, Y. Kim, and Y. Park, Quasinormal modes from potentials surrounding the charged dilaton black hole, Eur. Phys. Jour. C 58617 (2008)

[30] S. Fernando, Greybody factors of charged dilaton black holes in $2+1$ dimensions, Gen. Rel. Grav. 37461 (2005)

[31] S. Fernando, D. Krug, and C. Curry, Geodesics in static charged black holes in 2+1 dimensions, Gen. Rel. Grav. 351243 (2003)

[32] S. Chandrasekhar, The Mathematical Theory of Black holes, Oxford University Press, UK (1992) 